Article publié dans la revue Espaces et Sociétés n¹43 Les territoires de I'informel, décembre 2010, p.63-79.

Version de l'auteur

Valérie Clerc, Chercheur et responsable de I'Observatoire urbain du Proche-Orient Institut français du Proche Orient - Ifpo - Damas - Syrie, v.clerc@ifporient.org

\title{
Du formel à I'informel dans la fabrique de la ville Politiques foncières et marchés immobiliers à Phnom Penh
}

\section{Résumé}

Les activités informelles participent pour une large part à la production et à la configuration des espaces urbains, notamment dans les quartiers informels qui abritent le tiers des urbains de la planète. Ces quartiers ne sont cependant pas exclusivement produits par des pratiques informelles. Les politiques publiques et les pratiques légales et institutionnelles jouent un rôle majeur dans leur configuration. Elles orientent les pratiques informelles, déterminent l'emplacement des quartiers et participent à leur production. Comment les pratiques informelles fabriquent-elles la ville en interaction avec les pratiques formelles? L'analyse des politiques publiques de reconstruction et des marchés fonciers et immobiliers des quartiers informels à Phnom Penh révèle une production urbaine à l'articulation des politiques publiques, des pratiques encadrées et des pratiques informelles.

Mots clefs : Quartiers informels, marchés fonciers et immobiliers, Phnom Penh, Damas, Beyrouth

\section{Abstract}

The informal activities contribute in large part to the development and configuration of urban spaces, particularly in the informal settlement areas that provide a habitat for a third of the urban population on Earth. However, these districts are not produced exclusively by informal practices. Public policies together with legal and institutional practices play a major role in these configurations. They affect the informal practices, determine the location of the settlements and contribute to their construction. How do these informal practices, in interaction with the formal practices, create the city? The analysis of the public policies on reconstruction and the land and housing markets of the informal settlement areas in Phnom Penh reveals an urban production at the juncture of the public policies, the supervised practices and the informal practices.

Key words : Informal settlements, land and real estate markets, Phnom Penh, Damascus, Beirut 
Les activités informelles participent pour une large part à la production des espaces urbains. On peut prendre à témoin l'importance des quartiers dits informels dans le monde : un milliard d'habitants en ce début de XXI siècle, la moitié des habitants des villes en développement, le tiers des urbains de la planète (UN-Habitat 2003). Parfois considérés comme une production marginale (seuls $15 \%$ à $20 \%$ des habitants à Beyrouth ou Phnom Penh), ils couvrent cependant bien souvent la majorité, voire la quasi-totalité des villes (en Éthiopie par exemple, Davis 2005).

L'adjectif « informel » n'est qu'un des termes utilisés pour qualifier ces quartiers qu'on appelle irréguliers, précaires, sous-intégrés, illégaux, taudis ou, selon les villes, bidonvilles, favelas, slums, barriadas... La diversité de ces dénominations reflète la difficulté à définir ces espaces, jusque pour les experts internationaux réunis par l'ONU-Habitat (le programme des Nations unies pour les établissements humains) en 2002 à I'occasion de la définition des Objectifs de développement du millénaire « Un bidonville ou taudis (s/um) est une zone d'habitation contiguë où les habitants sont dotés de logements et de services de base insuffisants. Le bidonville ou taudis n'est souvent pas reconnu ou pris en compte par les autorités comme une partie intégrante et équivalente de la ville. » La réunion d'experts qui a adopté cette définition a recommandé le suivi de I'amélioration de ces quartiers à travers cinq composantes qui caractérisent le bidonville : «insécurité de la tenure, accès insuffisant à l'eau, accès insuffisant à l'assainissement et aux autres infrastructures de base, qualité de la structure du logement insuffisante, surpeuplement » (UN-Habitat 2003).

Cette définition et ces critères ne font pas directement référence à l'informalité des pratiques qui prévalent lors de la formation de ces quartiers. Ils laissent cependant entendre que leur formation s'est faite en dehors du contexte institutionnel et légal qui encadre les activités d'aménagement urbain, de construction ou d'urbanisme : insuffisance des services de base ou de la qualité de la construction (par rapport à une norme), espaces non reconnus par les autorités (conçus hors de la sphère institutionnelle), insécurité de la tenure (non consolidée par des titres légaux). Ils suggèrent ainsi que leurs bâtisseurs ont contourné, évité ou détourné les contraintes du contexte formel.

Produits par des activités informelles, ces quartiers ne le sont cependant pas exclusivement. Ils sont plutôt produits par des pratiques que I'on pourrait situer dans un «entre-deux » du formel et de l'informel. Certes, si I'on considère que l'irrégulier est ce qui n'est pas régulier et que l'informel est ce qui n'est pas formel, être à moitié légal, c'est ne l'être pas, et il suffit d'un peu d'informel pour ne plus être formel. Cependant, en avançant l'idée d'un « entre-deux», cet article met en avant la complexité de pratiques que l'on a tendance à définir en opposition, ou au moins par rapport, à l'espace institutionnel et légal. Or, le contexte et les pratiques légales et institutionnelles jouent un rôle majeur dans la 
configuration des quartiers informels. Ils orientent les pratiques informelles, déterminent l'emplacement des quartiers et participent à leur production. La question se décale alors de : «comment les activités et pratiques informelles contribuent-elles à la fabrication la ville ? » à « comment fabriquent-elles la ville en interaction avec les pratiques institutionnelles formelles ? ». Où se situent les interactions ? Quelles sont les pratiques intermédiaires ou spécifiques de la construction des quartiers informels?

Pour considérer cette question, nous aborderons dans une première partie les quartiers informels comme un revers urbain des politiques publiques foncières et d'urbanisme. D'une part, le cadre légal et règlementaire des villes est en constante construction et des espaces deviennent plus ou moins irréguliers au gré de son évolution. D'autre part, l'emplacement et le développement des quartiers informels sont largement déterminés par les histoires foncières et urbanistiques et par les politiques publiques d'habitat et de l'immobilier (cf. point I). Dans une seconde partie, nous verrons comment formel et informel sont imbriqués dans les marchés fonciers et immobiliers, comment au sein même des quartiers informels, les institutions et procédures légales interviennent dans la vie quotidienne des habitants et dans les événements qui participent à la création et au développement de ces quartiers. (cf. point II).

Les réflexions présentées dans cet article sont fondées sur des travaux de recherche menés successivement à Beyrouth (Liban), Phnom Penh (Cambodge) et Damas (Syrie). Les idées et questions théoriques présentées ont émergé de la confrontation de ces trois terrains. Pour illustrer le propos, l'article se concentre sur le cas révélateur de Phnom Penh et de ses marchés fonciers et immobiliers, mais l'analyse se réfère également à Beyrouth et Damas.

\section{Les quartiers informels comme revers urbain des politiques publiques}

Trois principaux types d'irrégularités caractérisent les quartiers informels. Les infractions aux règles de la construction existent de façon diffuse dans les villes, mais sont généralisées dans ces quartiers. L'irrégularité vis-à-vis des règles d'urbanisme leur est spécifique. L'occupation de terrains d'autrui concerne bon nombre de ces quartiers. Combinant ces irrégularités, les quartiers informels font généralement l'objet d'une distinction entre quartiers illégaux (les habitants occupent des terrains d'autrui) et lotissements irréguliers (les occupants ont la propriété du terrain, mais n'avaient pas le droit d'y bâtir).

Les quartiers informels se développent quand l'urbanisme planifie et règlemente l'espace à bâtir et la construction de façon insuffisante ou inadéquate, quand le marché formel ne propose pas de logement bon marché, quand l'État n'offre pas d'alternative pour l'accès au logement des pauvres. Le propos ici n'est pas de montrer l'impuissance des autorités à éviter ou à endiguer le développement de la ville informelle, ni 
de confirmer l'impact du retrait de l'État sur le développement de ces quartiers (UN-Habitat 2003), mais d'identifier différentes facettes du rôle, direct ou indirect, des politiques publiques (foncières, d'urbanisme et d'habitat entre autres) dans la formation des quartiers informels et de mettre en lumière les mécanismes par lesquels elles contribuent, « de l'extérieur », à leur production.

\section{Les quartiers informels en miroir de la construction du droit foncier}

Première dépendance des quartiers informels à l'égard du contexte institutionnel, le caractère illégal ou irrégulier des quartiers n'est pas stable dans le temps. Il dépend de l'évolution des lois et des règlements d'urbanisme, suivant la chronologie des politiques urbaines et foncières, et en particulier de celle du droit foncier et de la propriété.

Le Cambodge offre un cas d'école ${ }^{1}$. Là, la réapparition des quartiers informels suit exactement la reconstruction du cadre légal et règlementaire et la réintroduction progressive de la propriété foncière privée. Le Cambodge est passé d'une collectivisation totale sous le régime des Khmers rouges (1975-1979) à une économie de marché depuis 1993. En avril 1975, lors de leur accession au pouvoir, les Khmers rouges, groupe communiste proche de l'idéologie maoïste et imposant la vie agraire, ont totalement vidé les villes de leurs habitants en quelques jours et la propriété a été abolie. En 1979, à la chute de ce régime, l'occupation vietnamienne qui lui a succédé a appliqué au Cambodge un régime socialiste : la terre appartenait à l'État et les habitants avaient seulement un droit de jouissance et de succession sur des terres d'habitation sur lesquelles ils devaient obtenir l'autorisation de résider. Pendant les années 1980, les habitants se sont réinstallés en ville: dans les constructions vides, sur les toitures terrasses des immeubles et sur des terrains disponibles, entre les bâtiments ou sur le domaine public, comme sur les bas-côtés des routes et des voies de chemins de fer ou sur les berges des lacs et des rivières. La distinction à cette époque se faisait moins entre des habitants de quartiers formels et informels qu'entre les habitants autorisés par les autorités à résider quelque part et les autres.

La période 1989-1993 marque un tournant dans I'histoire urbaine du Cambodge. Politiquement, la période scelle le retour de la souveraineté nationale: départ des troupes vietnamiennes, réconciliation nationale, réouverture du pays et retour de la royauté. Dans le même temps, la propriété foncière privée est progressivement réintroduite. En 1989, une nouvelle constitution et des textes de lois autorisent la propriété privée foncière et organisent la redistribution générale des terres occupées. Tous les chefs de familles ont pu cette année-là faire la demande à l'État de la propriété de la résidence qu'ils occupaient. Les terrains non appropriés restaient propriété de I'État. Environ 4,5 millions de demandes de propriété ont été faites, dans un pays d'environ huit millions d'habitants.

\footnotetext{
${ }^{1}$ Sauf mention contraire, les données sur le Cambodge sont issues d'une recherche réalisée entre 2003 et 2005 sur les marchés fonciers et immobiliers des quartiers informels à Phnom Penh (Clerc et Rachmulh 2008b).
} 
En dépit de la mise en place du cadastre à partir de 2002, seules quelques centaines de milliers de demandes sont devenues des titres de propriété (le processus d'enregistrement définitif est compliqué et cher au regard de la valeur du bien). En 1993, la nouvelle constitution promulgue l'abandon de l'économie socialiste, l'adoption de l'économie de marché et la réinstauration du droit de propriété privée.

La période 1989-1993 est aussi celle de l'afflux de populations et de capitaux extérieurs. La reconstruction de la capitale est largement financée par l'aide internationale et les investisseurs reviennent. D'importants mouvements de population induisent une forte demande de logements. Les familles cohabitant jusqu'alors dans des mêmes maisons pour des raisons de sécurité cherchent un nouveau logis (la propriété d'un logement était attribuée à une seule famille). D'autres doivent quitter les bâtiments publics qu'elles occupent. Mais surtout, la capitale se gonfle, entre 1990 et 1993, des militaires démobilisés, des déplacés et des réfugiés de retour des camps de Thaïlande ainsi que de nombreux ruraux chassés par l'insécurité, les mines, la pauvreté et la poursuite des combats dans les campagnes. Deux conséquences principales de ces évolutions furent I'amplification du nombre de transactions foncières et immobilières (et de la spéculation) et la réapparition progressive des quartiers informels.

Contrairement à de nombreuses villes dans lesquelles les quartiers informels existent depuis des dizaines d'années, à Phnom Penh, les quartiers informels d'avant-guerre ont disparu après que la ville a été vidée de ses habitants en 1975 . La réapparition des quartiers informels correspond à la réinstauration de la propriété privée et à la distribution des terrains de résidence en 1989 qui ont abouti à l'apparition d'occupants sans droits : ceux qui ont tardé à déposer ou confirmer une demande de propriété et surtout ceux qui n'ont pu en faire la demande parce que l'espace qu'ils occupaient n'était pas appropriable, comme les terrains et bâtiments publics. En délivrant pendant la décennie précédente des autorisations d'occupation sur ces terrains, les autorités locales avaient ainsi contribué à l'émergence de ces zones informelles d'habitat (Carrier 2003). L'afflux de population et la forte demande de logements ont fait le reste. Les nouveaux arrivants se sont installés comme les précédents sur des terrains libres. Mais, malgré la validité jusqu'en 2001 de la loi foncière de 1992 qui permettait de devenir propriétaire d'une terre occupée pendant cinq ans de façon pacifique, continue, paisible et publique, les parcelles vacantes sont devenues rares et les nouveaux arrivants n'y ont pas eu accès. Ils se sont donc installés sur des terrains qui n'étaient pas appropriables et, souvent moyennant un paiement informel des nouveaux arrivés aux autorités locales, des quartiers dits alors « de squatters » se sont développés.

Le nombre d'habitants des quartiers informels n'a pas cessé d'augmenter depuis, suivant le même processus. La promulgation en 2001 de l'actuelle loi sur la propriété immobilière, qui marque la fin de la période transitoire 


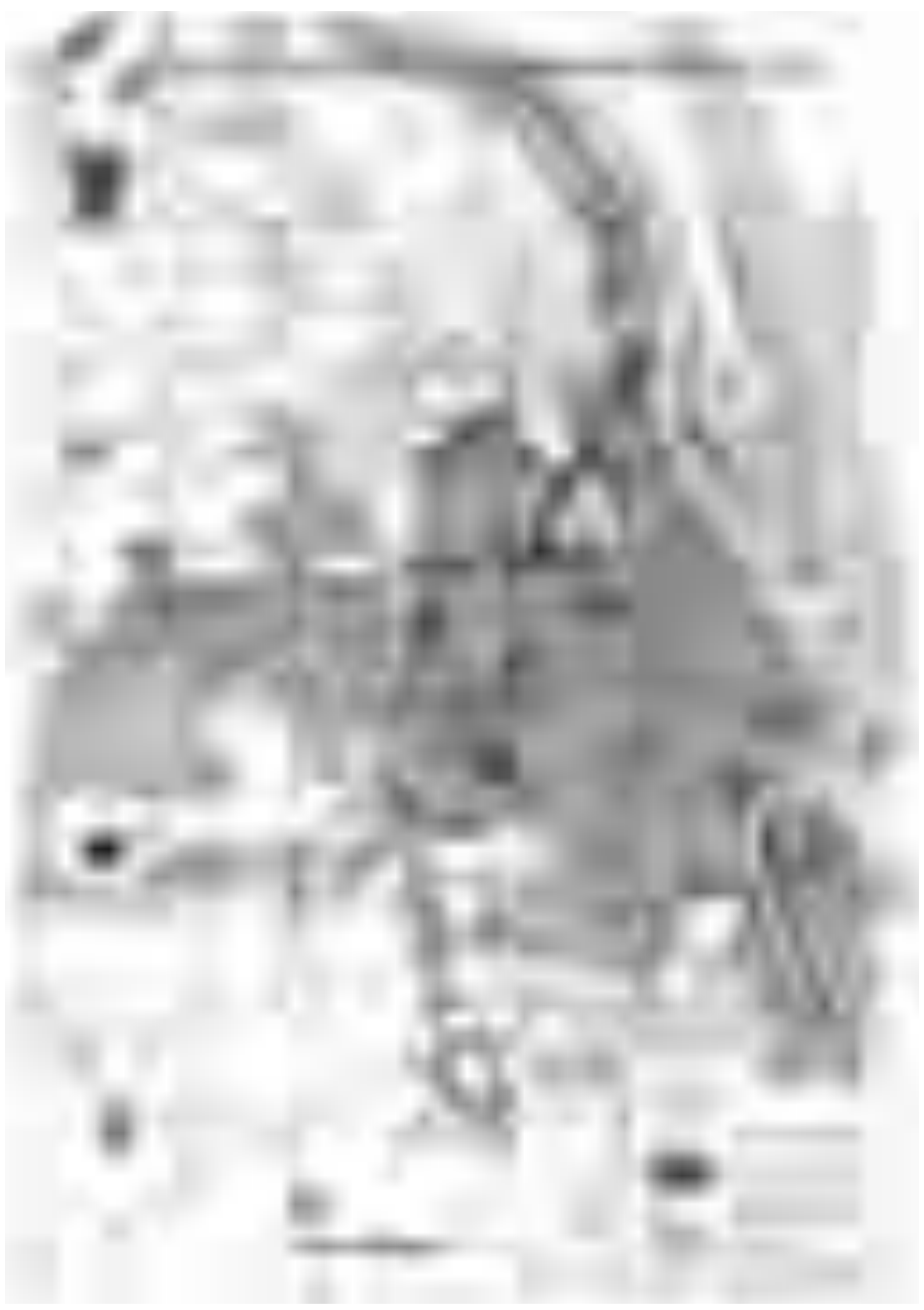

Carte des quartiers informels de Phnom Penh en 2005 
et met fin à la possibilité de devenir propriétaire par l'occupation, n'a pas interrompu ce mouvement. La possibilité d'attribuer des concessions sociales aux pauvres, que cette même loi instaure - fournir gratuitement, sous certaines conditions, un terrain aux familles qui n'ont pas les moyens d'en acheter un, sur des lotissements réalisés sur le domaine privé de I'État - , a été très peu appliquée. Dix ans après le retour à l'économie de marché, le début de la reconstruction du cadre législatif et la réintroduction de la propriété foncière privée, les quartiers informels ont réapparu. Ils abritent 220000 habitants recensés et environ 75000 locataires non recensés, pour une ville d'1,2 million d'habitants, soit $18 \%$ des ménages recensés et près du quart de la population si l'on compte les locataires (voir carte).

\section{Les quartiers informels en revers de l'urbanisme}

Deuxième dépendance des quartiers informels à l'égard du contexte institutionnel, certains terrains sont plus susceptibles que d'autres d'être occupés illégalement. Ils sont déterminés, en creux, par les politiques publiques foncières et d'urbanisme. Les quartiers informels se développent là où des règlements inadéquats incitent à leur contournement, là où les procédures institutionnelles et légales ont des «ratés », là où des propriétaires indifférents, des procédures d'urbanisme ralenties ou des conflits fonciers rendent des terrains plus vulnérables que d'autres à I'occupation.

Cet aspect est particulièrement mis en évidence dans les cas moyenorientaux. Beyrouth offre deux exemples. En banlieue sud-est, politiques urbaines et lois successives ont orienté l'activité des lotisseurs clandestins (Fawaz 2004). En banlieue sud-ouest, les quartiers illégaux se sont presque exclusivement développés sur des espaces où l'urbanisme et les politiques foncières avaient rendu les terrains peu intéressants à défendre contre l'occupation illégale: grandes parcelles non loties, souvent détenues en indivision, parcelles rendues inconstructibles par la réglementation, terrains situés dans l'emprise de remembrements non aboutis, à la suite de plans d'urbanisme non appliqués ou de conflits fonciers. Les quartiers informels se localisent exactement sur ces terrains, où se concentrent les lenteurs et les manques urbanistiques, les superpositions de droits et les conflits du droit foncier (Clerc 2008a).

À Damas, où environ $40 \%$ de la population habite des quartiers informels, la loi elle-même a largement contribué à la formation et au développement de ces quartiers. Elle a en particulier orienté leur localisation vers des terrains expropriés ou en voie de l'être. La loi d'expropriation 60 de 1979, en vigueur jusqu'en 2000, a imposé que toutes les extensions urbaines des capitales de province soient loties par des institutions publiques. Tous les terrains devaient y être expropriés au profit des municipalités, à leur valeur agricole. Leurs propriétaires se sont alors empressés de vendre ces terrains à leur valeur urbaine et des constructions illégales s'y sont rapidement développées (Baridi 2005). 
Le développement des quartiers informels apparaît ainsi très dépendant des heurts et manques du contexte institutionnel et légal. Ces heurts ont influencé les propriétaires, qui ont, passivement ou activement, abandonné ou vendu leurs terrains aux lotisseurs et occupants irréguliers. Les quartiers informels manifestent dès lors ce qu'on peut considérer comme un revers de l'urbanisme et des politiques foncières (Clerc 2008a).

\section{Défauts et défaut de politiques publiques : le nœud foncier}

Dans les deux cas moyen-orientaux et cambodgien, les quartiers informels ont émergé dans les insuffisances, les ombres et les défauts des politiques publiques foncières et d'urbanisme. Ces insuffisances sont régulièrement multipliées par l'absence ou la faiblesse des politiques de protection sociale - nombreux sont ceux qui doivent vendre leur logement formel pour faire face à des dépenses de santé ou la perte d'un emploi et deviennent locataires ou occupants de quartiers informels- et les politiques d'habitat. Ces dernières, insuffisamment articulées avec le contexte économique, social et politique, peuvent même avoir l'effet inverse de celui escompté. Des programmes de régularisation des quartiers informels participent ainsi à la propagation de l'informalité : faute d'une caractérisation adéquate de ces quartiers (Smolka et Biderman 2009) ou d'une prise en compte suffisante des dynamiques nationales et locales de leur formation (Hurchzermeyer et Karam 2006).

Les politiques de régularisation entrent parfois en concurrence avec d'autres politiques, comme l'appui au développement des investissements, source de contradictions dans l'action publique. Le foncier est un nœud principal du problème. L'exemple du Cambodge est là encore percutant. Depuis 1993, le gouvernement a expérimenté plusieurs politiques pour résorber les quartiers informels de Phnom Penh, jusqu'en 2003 où il s'est finalement engagé à les régulariser tous. En appui à ces politiques, la coopération internationale a financé de nombreux projets d'équipement des quartiers ainsi que plusieurs études sur l'amélioration de la sécurité foncière (Rabé 2004, Payne 2005). Or quelques années après, la régularisation annoncée n'est pas formalisée et une politique concurrente en entrave la réalisation. En effet, alors que le gouvernement affiche sa résolution à agir pour que les plus pauvres conservent leurs droits fonciers, il engage en même temps les investisseurs à développer les terrains occupés des domaines publics et privés de l'Etat du centre ville. Ces investisseurs ambitionnent d'en déloger les habitants, qui eux réclament de rester sur place, comme promis par les autorités. Le gouvernement louvoie, encourage d'une main les communautés des quartiers informels, mais soutient les investisseurs de l'autre. Les contradictions sont gérées au cas pas cas, terrain par terrain, par la négociation et dans des rapports de force (Clerc 2005). Hormis une opération en centre-ville ayant relogé sur place plus de 1700 familles (Boreï Keyla), la pratique est au relogement en lointaine banlieue. Les plus pauvres ne résistent pas à cet éloignement de leur lieu de travail et reviennent s'installer dans d'autres quartiers informels centraux, qui 
croissent (Falavier 2002). L'aide internationale renforce cette tendance en mettant la priorité de ses financements dans la sécurisation de la propriété (cadastre) au détriment de la lutte contre la pauvreté urbaine, en dépit d'une référence affichée aux Objectifs du millénaire (Huybrechts 2008). Les politiques de résorption des quartiers informels butent ainsi sur les mêmes obstacles que ceux qui ont favorisé le développement de ces quartiers : des dynamiques de marchés fonciers et immobiliers induites et/ou non maîtrisées par les politiques publiques foncières, d'urbanisme, sociales ou d'habitat.

\section{Intrications des marchés fonciers et immobiliers des quartiers formels et informels}

Construits en étroite interaction avec le cadre institutionnel et les politiques publiques, qui participent à leur développement «de l'extérieur », les quartiers informels sont aussi le lieu de la construction de normes propres et de règles spécifiques qui façonnent ces quartiers « de l'intérieur». Parler d'informalité des pratiques ne signifie pas que les activités soient caractérisées par une absence de règles: la non conformité aux règles formelles se double dans ces quartiers de la conformité à des règles informelles (Razzaz 1996). Considérés comme des lieux à la marge, «ban-lieu» (Agier 1999), ces quartiers ont leurs dynamiques propres. Les modalités informelles de production des lieux sont variées et loin d'être marginales (Vallat et alii, 2008). L'observation de la production matérielle non planifiée de la ville fait apparaître des compétences en situation, des savoir-faire, des innovations. Elle a permis l'identification des règles, des normes sociales et des valeurs prévalant dans la création des quartiers populaires (Berry-Chikraoui et Deboulet 2000).

Cette production interne n'est toutefois pas le seul fait des pratiques informelles. Malgré la fragmentation spatiale qui les sépare physiquement de la ville, les quartiers informels sont inscrits dans ses marchés fonciers et immobiliers et sont liés à eux dans un continuum formel/informel. Les univers formels et informels sont indissociables dans la fabrication des quartiers informels. Le formel n'est pas regardé ici comme l'espace contre lequel l'informel se construit, dont on se détache, mais comme une présence qui participe à la production de ces quartiers. Les dynamiques formelles y agissent en articulation avec les pratiques informelles, comme parfois dans les quartiers formels.

C'est justement une des raisons pour lesquelles il est difficile de définir précisément les quartiers informels. À Phnom Penh, aucune définition officielle ne permet de caractériser cet habitat. Les rapports d'expertise parlent de «groupes d'unités de logements qui se développent de façon non systématique sur les terrains vacants publics ou privés » (Green 2004). La limite exacte entre formel et informel y est difficile à appréhender. Dans ces quartiers, même si le plus généralement ce n'est 
pas le cas, il arrive que les habitants aient des titres, aient accès aux services et infrastructures publics, habitent dans des maisons en dur ou soient assez riches. Les habitations ne sont généralement pas organisées suivant un plan orthogonal, mais il y a des exceptions. Enfin, I'habitat est généralement beaucoup plus dense que dans le reste de la ville. Bien qu'imparfaite, l'approche par la morphologie urbaine et la densité est donc souvent adoptée, faute de mieux et parce qu'elle permet leur localisation sur photo aérienne (voir illustration). En revanche, malgré cette difficulté à caractériser précisément ces quartiers, sur le terrain, c'est la prédominance de l'informalité qui les caractérise. Les habitants se perçoivent clairement vivre dans des quartiers informels, « pas normaux, risqués » dit une habitante à Phnom Penh. Les acteurs de l'urbanisme les reconnaissent comme tels dans la mise en place des politiques urbaines. Les ONG et réseaux internationaux participent également à cette distinction en suscitant la création de communautés de pauvres dans ces quartiers (pour créer des groupes d'épargne et mettre ces quartiers en réseau international), comme I'ont fait au Cambodge Asian Coalition for Housing Rights (ACHR) ou Shack/Slum Dweller international (SDI) depuis les années 1990 (Clerc 2005). L'observation de l'intrication entre le formel et l'informel dans la dynamique de production de l'espace urbain informel contribue donc à éclairer les raisons pour lesquelles les quartiers informels sont difficiles à définir de façon rigoureuse, malgré la perception claire que tous en ont.

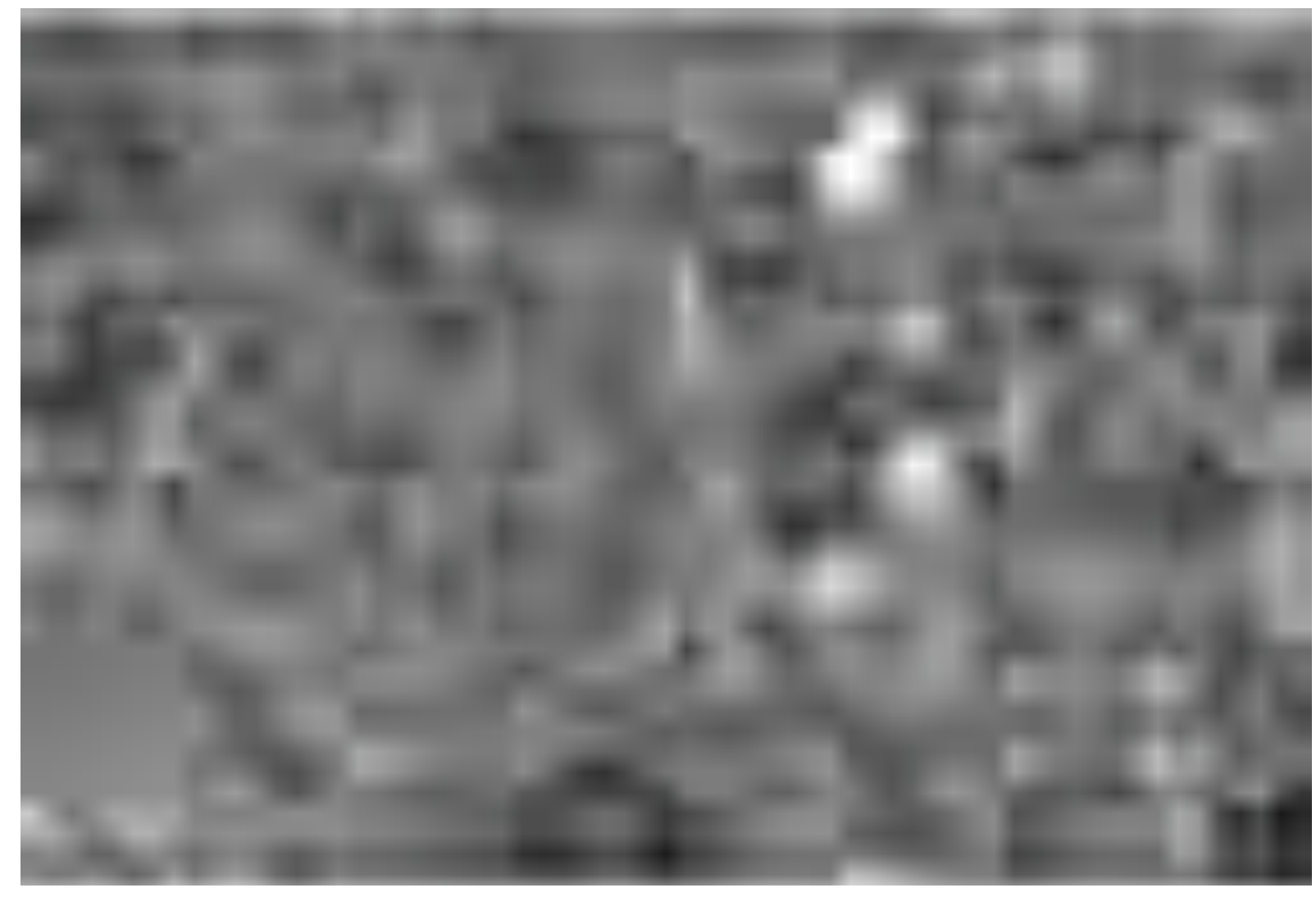

Tissus urbains formels et informels près du Boeng Salang, Phnom Penh Image satellite Google Earth 2005 
Les marchés fonciers et immobiliers des quartiers informels de Phnom Penh partagent de nombreuses caractéristiques avec ceux des logements formels : une part importante de l'accès à la terre et au logement s'est faite à titre gratuit après 1979, la marchandisation est aujourd'hui quasi généralisée et les transactions y sont informelles à une écrasante majorité. Ils s'en distinguent d'une façon relative, par une plus grande informalité des procédures, par des prix inférieurs, qui reflètent une plus grande insécurité foncière et un moins bon accès aux infrastructures, par la présence d'une plus grande proportion de pauvres et surtout par une quasi impossibilité d'être régularisé suivant la législation en cours (les propriétaires ne pourraient pas commencer une procédure de demande de titre foncier). Mais les limites ne sont pas étanches. Les marchés formels et informels se recoupent pour ce qui est de leur clientèle, de leur prix, de leur équipement; ils se rejoignent sur les procédures de transactions, la progressivité de la construction, la présence de subventions publiques.

\section{Des ménages oscillant du formel à l'informel}

En premier lieu, de nombreux parcours résidentiels font fi des frontières entre quartiers formels et informels. À Phnom Penh (comme également à Damas), les habitants déménagent d'un type de quartier à l'autre, dans un espace urbain plus large que celui des quartiers informels, bousculant l'idée d'un partage figé de la ville entre deux types d'habitants. Les nouveaux installés dans les quartiers informels de Phnom Penh ont toujours un parcours résidentiel urbain avant d'y investir (location, hébergement), dont les étapes se font régulièrement dans les quartiers formels.

L'installation dans un quartier informel constitue généralement elle-même une étape dans un parcours résidentiel urbain plus long, stable, ascendant, descendant ou oscillant. Beaucoup passent d'un logement à l'autre dans les quartiers informels (hébergement, location, achat, décohabitation, division d'un lot, appropriation d'un nouveau terrain, éviction d'un autre quartier). Mais les exemples de passages formel/informel dans les parcours sont fréquents. Certains s'installent dans ces quartiers avant d'accéder à un quartier formel (nouveau travail plus rémunérateur, épargne suffisante). D'autres à l'inverse y arrivent après avoir dû quitter leur logement dans un quartier formel (appauvrissement, enfants décohabitant après leur mariage). D'autres enfin oscillent du formel à l'informel (les locataires notamment).

\section{Dynamiques liées et superposition de segments de marchés}

Les marchés immobiliers des quartiers informels et formels de Phnom Penh sont liés. Lorsque le marché formel produit davantage de logements pour les bas revenus, la croissance de ces quartiers diminue ou stagne (comme cela s'est produit par exemple à Phnom Penh en 2004). Face à la demande locative, l'adaptation a été rapide tant dans les quartiers informels que formels, lorsque de quelques milliers dans les années 1990, les ouvriers du textile sont passés à plus de 360000 en 2008 à Phnom 
Penh (International Labour Organisation 2008). Dans les quartiers informels, où les locataires forment $25 \%$, ils sont parfois plus de $70 \%$ à proximité des industries (construction de nombreuses maisons locatives, chambres à louer chez tous les ménages).

L'analyse des produits achetés ou construits montre une superposition des segments bas du marché formel et du segment haut du marché de l'informel. Des trois produits de logement généralement proposé par le marché formel - maisons individuelles, terrain à bâtir et compartiments (constructions allongées mitoyennes de 4 mètres de façade sur 8 à 24 mètres de profondeur, sur un ou plusieurs niveaux)-, ces derniers sont de loin les plus nombreux. Leur prix varie de $4000 \$$ à $180000 \$$, avec un prix moyen de $20000 \$$ (Danchin 2005). Les quartiers informels de Phnom Penh recèlent un large éventail de constructions, de la cabane en paille (souvent moins de $10 \mathrm{~m}^{2}$ ) à la maison en brique à étage de type compartiment. Les prix varient de $10 \$$ à $10000 \$$ (2004). Les maisons de moins de $100 \$$ sont petites, en paille, sans porte ni fenêtre, ni eau, toilettes ou électricité, en zone inondable et sur terrain squatté. Le prix des maisons de $100 \$$ à $2000 \$$, les plus fréquentes, varie suivant l'emplacement et la qualité (bois/tôle, bois/paille, un ou deux niveaux). Les maisons de $2000 \$$ à $10000 \$$, de bonne qualité (bois, maçonnerie, eau, électricité, toilettes), sont bien situées et d'une relative sécurité foncière. Certaines ont donc des prix supérieurs aux prix des compartiments les moins chers construits dans le marché formel en lointaine banlieue $(4000 \$)$. Comme dans le marché formel, il y a un marché spéculatif des terrains dans les quartiers informels, les prix ayant parfois doublé entre 2000 et 2004 . Des ventes entre $7 \$ / \mathrm{m}^{2}$ et $50 \$ / \mathrm{m}^{2}$ dans des quartiers informels bien situés et estimés bénéficier d'une bonne sécurité foncière dépassent largement les prix du marché formel en périphérie ( 1 à $3 \$$ le $\mathrm{m}^{2}$ ).

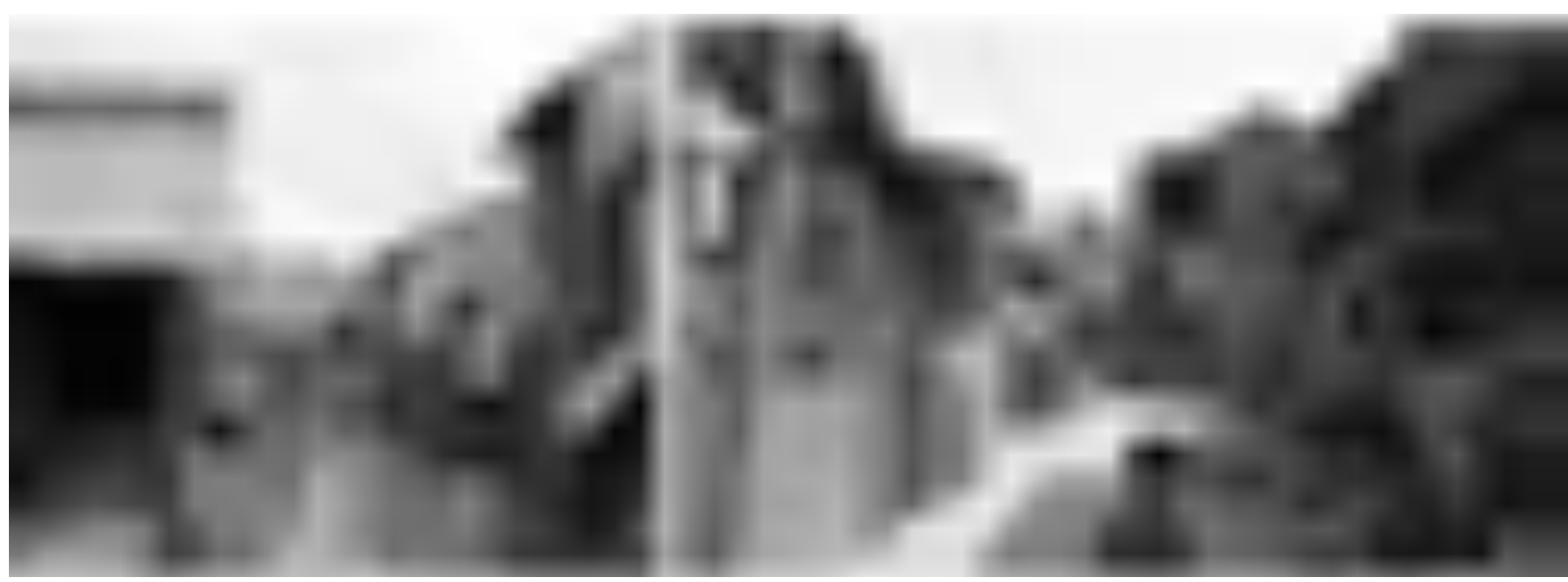

Les quartiers informels de Phnom Penh recèlent un large éventail de constructions, de la maison en paille (à gauche, quartier informel de Bassac au centre ville) à la maison en brique et bois à étage de type compartiment (à droite, quartier informel de Prek Tanou au sud). Photos V.Clerc 2005 
Recoupements des pratiques constructives et des accès aux infrastructures

À Phnom Penh, certaines pratiques sont communes aux quartiers formels et informels, comme la construction progressive de la maison. Sur les terrains squattés des quartiers informels, les plus pauvres construisent progressivement des lits abrités $(10 \$$ à $25 \$)$, puis des cabanes en paille ( $75 \$$ à $250 \$)$, plus rarement des maisons en bois et tôle (300 à $600 \$$ ). Sur les terrains achetés, se construisent, souvent progressivement, des maisons de deux niveaux en bois et tôle $(700 \$$ à $1600 \$)$ ou en maçonnerie et bois $(1800 \$$ à $5000 \$)$. La construction progressive des habitations est adaptable à l'irrégularité des revenus. Ce processus n'est pas différent de celui qu'on observe dans les quartiers formels éloignés du centre (les villages alentours, les sites de réinstallation), où habite aussi une population pauvre.

Le secteur formel des infrastructures n'est pas absent des quartiers informels, où l'on constate une coprésence des accès formels et informels à l'eau et à l'électricité. Malgré un mauvais accès global aux services, nombre de ménages des quartiers informels sont abonnés à la Régie des eaux de Phnom Penh ( $17 \%$ en 1999) et à Électricité du Cambodge (33\% en 2004). Les autres ont des branchements illégaux ou ont affaire à des particuliers revendeurs (ils achètent l'eau par jarres, bidons ou bouteilles ; ils achètent leur électricité à d'autres habitants, ou utilisent une batterie), tandis qu'environ $15 \%$ n'ont pas accès aux infrastructures (ils tirent l'eau des puits, mares et rivières; ils s'éclairent avec des lampes à pétrole, des lampes à huile ou à la bougie), toutes situations qui ne sont pas inconnues des quartiers formels (dans les villages et les sites de réinstallation notamment). L'accès aux services publics dans les quartiers informels se renforce, notamment en raison des multiples petits projets d'assainissement, de drainage et de chemins d'accès réalisés par les ONG et I'ONU-Habitat et d'une politique de distribution de l'eau de la ville aux quartiers pauvres, qui se poursuivent aujourd'hui.

Partage du même accès gratuit originel

Les modes de financement du logement dans les quartiers informels sont variés et recoupent ceux des quartiers formels. L'apport personnel vient de l'épargne (certains financent ainsi entièrement leur logement), d'autres revendent un bien précédent ou bénéficient de dons de parents plus fortunés ou de connaissances. L'accès informel au crédit est contrasté. Beaucoup empruntent sans intérêt et sans délai fixe à la famille ou à des connaissances, quelques uns empruntent à des ONG (entre $8 \%$ par an et $3 \%$ par mois), d'autres utilisent les tontines. Enfin, le recours à l'emprunt à des usuriers est fréquent (de $6 \%$ à $30 \%$ par mois) et certains empruntent même toute la somme à des taux usuraires. Les modes de financement sont combinés, associant parfois emprunts sans intérêt et à 
taux usuraire, et paraissent dépendre moins des revenus des emprunteurs que des opportunités.

L'État joue un rôle a posteriori dans le financement du logement. Ceux qui peuvent revendre un bien obtenu gratuitement bénéficient de la subvention de fait de l'État qu'avait constitué le don des terres à leur occupant à partir de 1989. Ces ménages tirent les fruits d'une installation urbaine ancienne et de l'attitude du gouvernement qui encourage la compensation des ménages évincés. Tous n'ont pas un tel capital de départ. En 2003, la moitié des ménages cambodgiens habite un espace obtenu gratuitement, autant dans les quartiers informels que formels. L'accès à la terre à titre gratuit ne persiste aujourd'hui que par le squat du domaine public (bords des routes et des rails, berges des lacs et rivières). Dans certains quartiers très pauvres et excentrés, un contrat oral est passé avec les autorités locales selon lequel les habitants peuvent rester, mais de façon provisoire, et devront partir sans réclamer aucune indemnité le jour où on le leur demandera.

\section{Des procédures de transferts similaires}

Seuls les terrains ayant des titres de propriété définitifs peuvent faire l'objet d'une transaction foncière et immobilière suivant la procédure formelle à la Municipalité. Comme, en 2002, encore $90 \%$ des parcelles du pays sont détenues sans titres définitifs ${ }^{2}$, les transactions foncières suivent presque partout des procédures informelles, dont se distinguent peu les pratiques des quartiers informels. Acheteur et vendeur vont voir ensemble, avec des témoins, l'autorité locale qui rédige un contrat signé par toutes les parties. Les autorités contactées ne sont pas toujours les mêmes et les documents ne sont pas signés ou tamponnés de la même façon. Dans les quartiers formels, l'autorité contactée est fréquemment le chef de quartier, rarement mobilisé dans les quartiers informels, où il est plus souvent fait appel à un échelon administratif inférieur.

Dans les quartiers informels comme formels, la sécurité de la tenure ne repose donc pas sur la possession de titres fonciers définitifs, mais sur celle de titres fonciers intermédiaires, attestant d'un commencement d'enregistrement de la propriété foncière (demande d'enregistrement, accusé de réception de la demande, rapport d'investigation foncière, annonce de la date de la délimitation, copie du plan d'enregistrement, annonce de l'affichage public, rapport de justice, résultats de conciliation, jugement du tribunal, certificats...) ou sur celle de documents, officiels ou non, utilisés comme preuves de résidence : livret de famille, carnet de résidence, contrat de vente, livret d'épargne, justificatifs d'abonnement à l'eau ou l'électricité, autorisation de faire des réparations. Les titres fonciers intermédiaires sont majoritaires dans les quartiers formels et très minoritaires dans les quartiers informels, où les documents officiels de

\footnotetext{
${ }^{2}$ L'écrasante majorité des «propriétaires » cambodgiens ne détient que l'accusé de réception de la demande de reconnaissance de la propriété effectuée en 1989.
} 
résidence sont majoritaires dans certains quartiers et inexistants dans d'autres. Ainsi, la quasi-totalité des ménages n'a pas de titre foncier définitif et réalise des transactions foncières informelles, mais les habitations des quartiers informels se distinguent de celles des quartiers formels par leur quasi absence de titre foncier intermédiaires. Toutefois, la sécurité foncière dépend des titres détenus et de l'attitude des pouvoirs publics, mais surtout, elle dépend de la localisation des terrains occupés et des projets (public ou privés) prévus sur les sites. En période de boom foncier et immobilier, avec une sécurité foncière incertaine liée à la rareté des titres définitifs, le processus d'éviction des quartiers informels par le marché (Durand-Lasserve 2006) est susceptible de toucher également les quartiers formels populaires.

L'analyse des marchés fonciers et immobiliers des quartiers informels à Phnom Penh montre donc des similitudes et des superpositions avec les marchés formels, ainsi que la présence de pratiques formelles dans la fabrication des quartiers informels. Ces recoupements concernent certains segments du marché, mais pas toujours les mêmes, montrant l'interdépendance et la perméabilité entre les marchés fonciers et immobiliers formels et informels.

\section{Conclusion : Au delà des quartiers informels, l'accès à l'habitat et à la ville centre}

L'analyse des marchés fonciers et immobiliers des quartiers informels révèle une production urbaine à l'articulation des politiques publiques, des pratiques encadrées et des pratiques informelles. L'observation des modes de production, des parcours résidentiels ou des marchés fonciers dans ces quartiers incite à les analyser dans leur interaction avec le reste de la ville. Les considérer seuls, ou en opposition avec les quartiers formels, permet d'en comprendre les logiques internes et donc de mieux connaître la ville qu'ils fabriquent, mais ne permet pas de bien appréhender leur rôle et leur poids dans le puzzle urbain, ni les logiques et les échecs des politiques publiques qui $s^{\prime} y$ attachent. De ce point de vue, I'opposition quartiers informels/quartiers formels est peu efficace.

Tant l'importance numérique des quartiers informels que leurs intrications avec la ville formelle renforcent d'abord la nécessité d'analyser marchés et politiques du logement sur l'ensemble de l'agglomération, quartiers formels et informels confondus. Elles confirment ensuite l'importance de considérer l'informel, non pas comme un espace intégrable à un espace formel, bien souvent plus petit que lui, mais comme un espace de la ville avec ses difficultés à réduire et ses atouts - les savoir-faire de ceux qui les ont bâtis, ses capacités à rendre l'habitat accessible aux plus pauvres - à valoriser.

Elles incitent enfin à observer de concert les politiques publiques ayant des implications spatiales. La concurrence foncière peut être au cœur des contradictions de l'action publique. Cette concurrence et les évictions par 
le marché ne menacent pas seulement les quartiers informels, mais de plus en plus souvent des quartiers populaires centraux et péricentraux légaux. Cela pose la question du rôle de la légalité dans ces dynamiques, si quartiers légaux et quartiers illégaux subissent le même sort. L'insécurité immobilière (le risque d'être évincé de son logement) ne serait pas seulement fonction de l'insécurité foncière (liée à l'absence d'un titre foncier solide), mais serait de plus en plus attachée aux mécanismes des marchés d'investissement immobilier, mécanismes que ne corrigent pas les politiques publiques. Cette rivalité pour le foncier urbain central remet au cœur du débat la place des politiques publiques d'habitat et de lutte contre la pauvreté dans le développement de la ville.

\section{Références}

AGIER M. (1999), L'invention de la ville, Banlieues, townships, invasions et favelas, Paris, EAC, $176 p$.

BARIDI O. (2005), Périurbanisation des métropoles arabes, Paris, Université Paris 12 , thèse de doctorat, $231 \mathrm{p}$.

BERRY-CHIKRAOUI I., DEBOULET A. (dir.) (2000), Les compétences des citadins dans le monde arabe, Penser, faire et transformer la ville, IRMC, Karthala, Urbama, 406p

CARRIER A. (2003), Les marqueurs de propriété et leurs interactions dans la relation espace urbain/habitat à Phnom Penh, Réseau Asie, Communication au Premier Congrès du Réseau Asie, 24-25 septembre 2003, 13p.

CLERC V. (2008 a), Les quartiers irréguliers de Beyrouth, une histoire des enjeux fonciers et urbanistiques de la banlieue sud, Beyrouth, Institut français du Proche-Orient (Ifpo), 290p.

CLERC V. (2008 b), avec la collaboration de Rachmulh V., Les marchés fonciers et immobiliers des quartiers informels à Phnom Penh, Cambodge, Paris, ministère des Affaires étrangères, GRET, 176p.

CLERC V. (2005), « Les politiques de résorption des quartiers informels à Phnom Penh. Influence des organisations internationales et contradictions de l'action publique », Géocarrefour, volume 80, n³, p.183-196.

DANCHIN G. (2005), Le marché immobilier à Phnom Penh, Paris, Ministère des Affaires étrangères, $35 p$.

DAVIS M. (2006), Le pire des mondes possibles, de l'explosion urbaine au bidonville global, Paris, La découverte, 250p.

DURAND-LASSERVE A. (2006), « Market-driven eviction and displacement : implications for the perpétuation of informal settlement in developig countries » in HUCHZERMEYER M. et KARAM A., Informal Settlements, a Perpetual Challenge ? Cape Town, UCT Press, p.207-230.

FALLAVIER P. (2002), Analysis of the survey on impacts of relocation projects upon livehoods conducted from January to march 2002 in five resettlements sites in Phnom Penh, UN-Habitat, 44p.

FAWAZ M. (2004), Strategizing for housing: an investigation of the production and regulation of low-income housing in the suburbs of Beirut, MIT, PhD, 299p.

GREEN I. (2004), Preliminary slum improvement strategy, Municipality of Phnom Penh, Phnom Penh Urban Poverty Reduction Project, UN- Habitat/UNDP/DFID 15p. 
HUCHZERMEYER M. et KARAM A. (2006) (dir.), Informal Settlements, a Perpetual Challenge ?, Cape Town, UCT Press, 318p.

HUYBRECHTS E. (2008), «Phnom Penh, I'urbanisation au gré des marchés », Études foncières $\mathrm{n}^{\circ} 133$, mai-juin 2008, p.42-46.

INTERNATIONAL LABOUR ORGANISATION (ILO), (2008), Better factories Cambodia, Twenty first synthesis report on working conditions in Cambodia garnment sector, Kingdom of Cambodia, 12p.

PAYNE G. (2005), « Getting ahead of the game, a twin-track approach to improving existing slums and reducing the need for future slums », Environment \& Urbanization Vol17 No 1 avril 2005, p.135-145.

PIERDET C. (2008), « Marges aquatiques et politiques urbaines au centre de Phnom Penh (Cambodge) », Autrepart n45, p.123-136.

RABÉ P. (2004), Pro-Poor Urban Land Management and Housing Strategy, Policy implementation guidelines for the Municipality of Phnom Penh, Municipality of Phnom Penh, Phnom Penh Urban Poverty Reduction Project UN- Habitat/UNDP/DFID , 71p.

RAZZAZ O. (1996), « The informal sector and new institutionnalism : theoretical and policy implications », papier non publié, 23p.

SMOLKA M. et BIDERMAN C. (2009), « Measuring Informality in Housing Settlements: Why bother? », Land Line, Lincoln Institute for Land Policy, avril 2009, p.14-19.

UN-HABITAT (2003), The challenge of slums, Londres, Earthcan, 310p.

VALLAT C., SEMMOUD N, MONNET J. et LEFRANÇOIS D. (2008), «Constructions illégales, activités informelles, interstices urbains: la ville, indomptable territoire », Historiens-géographes, n403, p113-123. 